\title{
Fermion RG blocking transformations and conformal windows
}

\section{Cheng}

Department of Physics and Astronomy, University of California, Los Angeles

Los Angeles, CA 90095, USA

E-mail: darktree@physics.ucla.edu

\section{E.T. Tomboulis*}

Department of Physics and Astronomy, University of California, Los Angeles Los Angeles, CA 90095, USA

E-mail: tomboulisephysics.ucla.edu

\begin{abstract}
We explore fermion RG blocking transformations in LGT with the aim of studying IR behavior. In the case of light fermions the main concern is ensuring locality of any adopted blocking scheme. We study how an exact fermion blocking transformation may be constructed which is manifestly local in a general gauge field background. We also present an approximate, easily implementable scheme for combined fermion-gauge field blocking, which, as a first modest goal, may allow quick, inexpensive estimates of the location of conformal windows for various groups and fermion representations. We apply such a scheme to SU(2) and SU(3).
\end{abstract}

The XXIX International Symposium on Lattice Field Theory - Lattice 2011

July 10-16, 2011

Squaw Valley, Lake Tahoe, California

${ }^{*}$ Speaker. 


\section{Introduction}

In view of the LHC a lot of attention is currently being given to the phase structure of nonAbelian gauge theories with varying fermion content. For sufficiently large number of fermions asymptotic freedom (AF) and confinement are lost. It is generally believed that there is an intermediate range with an UV AF fixed point but no confinement; instead there should be an IR fixed point resulting in conformal IR dynamics. Exploring the dependence of these 'conformal windows' on the gauge group, the number of fermion multiplets and their representation, and the structure of the associated IR fixed points, i.e. their relevant directions and anomalous dimensions, is of central importance for informed model building "beyond the standard model".

Several approaches have been employed over the last few years in order to investigate these issues [1]. One popular method is to adopt some definition of a running 'coupling, e.g. the 'Schroedinger functional' coupling definition. Since any definition of a 'coupling' outside the perturbative regime is necessarily arbitrary, any such choice is scheme-dependent. Caution should then be exercised in interpreting results. Another approach is to compute the spectrum and/or some judiciously chosen physical observable and track their deformation as a function of the number of fermions and their masses. This should, in principle, yield an unambiguous outcome, but is clearly very expensive to carry out in practice to the degree of accuracy required for unambiguous results. Furthermore, it is not always clear what the expected behavior should be.

The above may be classified as indirect methods. A direct method for getting at the phase structure would be implementation of the Wilsonian Renormalization Group (RG), ie. RG blocking transformations for direct determination of the effective action RG flow and its fixed points. Conceptually, this is the most straightforward and unambiguous approach but challenging to set up in the presence of light fermions. Studies using the two-lattice matching MCRG method have recently appeared in [2].

\section{Fermion RG blocking}

In devising RG blocking transformations for light fermions the main concern is maintaining reasonable locality of the blocked action after each blocking step. This is a question of interest in it's own right apart from issues of practical implementation. For free fermions a blocked action can be defined along the lines below (eq. (2.3)-(2.4)) that can be shown to maintain locality [3]. This has been used to argue [4] the locality of determinant rooting for free fermions. This locality demonstration, however, cannot be extended in the presence of an arbitrary gauge field background.

To begin exploring this issue consider the Wilson operator in background gauge field $U$ :

$$
D_{0}(U)=1-\kappa M[U]
$$

where the only non-vanishing elements of matrix $\mathrm{M}$ are:

$$
M_{n(n+\hat{\mu})}[U]=\left(1-\gamma_{\mu}\right) U_{\mu}(n), \quad M_{n(n-\hat{\mu})}[U]=\left(1+\gamma_{\mu}\right) U_{\mu}^{\dagger}(n-\hat{\mu}) .
$$

A fermion RG blocking step specifies a transition from the fermion degrees of freedom on the original lattice $\Lambda$ to 'thinned-out' degrees of freedom on a lattice $\Lambda^{\prime}$. With $\Lambda$ a lattice of spacing 
$a$, let $\Lambda^{(1)}$ denote the lattice of spacing $2 a$. We take $\Lambda^{\prime}=\Lambda^{(1)} \cup S$, where $S$ is a subset of $\Lambda$ to be specified later.

We introduce variables $\Psi^{(1)}$ on $\quad \Lambda^{\prime}=\Lambda^{(1)} \cup S$ by

$$
\begin{aligned}
& \int D \bar{\psi} D \psi \exp \left(-\bar{\psi} D_{0} \psi\right) \\
= & \int D \bar{\psi} D \psi D \bar{\Psi}^{(1)} D \Psi^{(1)} \exp \left\{-\bar{\psi} D_{0} \psi-\alpha\left(\bar{\Psi}^{(1)}-\bar{\psi} Q^{\dagger}\right)\left(\Psi^{(1)}-Q \psi\right)\right\} .
\end{aligned}
$$

The rectangular matrices $Q: \Lambda \rightarrow \Lambda^{\prime}$ are given by

$$
\begin{aligned}
Q_{n m} \psi_{m} & =\psi_{n}+\zeta \sum_{\mu=1}^{d} M_{n(n+\hat{\mu})}[U] \psi_{(n+\hat{\mu})}+\zeta \sum_{\mu=1}^{d} M_{n(n-\hat{\mu})}[U] \psi_{(n-\hat{\mu})} \\
& \equiv\left(\delta_{n m}+\zeta \tilde{Q}_{n m}\right) \psi_{m} .
\end{aligned}
$$

Note that $\alpha \rightarrow \infty$ gives a $\delta$-function definition of the $\Psi^{(1)}$ 's, i.e. $\Psi_{n}^{(1)}=(Q \psi)_{n}$. Integrating out the original variables one has on the new lattice $\Lambda^{\prime}$ :

$$
\begin{aligned}
& \int_{\Lambda} D \bar{\psi} D \psi \exp \left(-\bar{\psi} D_{0} \psi\right) \\
= & \operatorname{Det} G^{-1} \int_{\Lambda^{\prime}} D \bar{\Psi}^{(1)} D \Psi^{(1)} \exp \left\{-\alpha \bar{\Psi}^{(1)} \Psi^{(1)}+\alpha^{2} \bar{\Psi}^{(1)} Q G Q^{\dagger} \Psi^{(1)}\right\}
\end{aligned}
$$

with

$$
G^{-1}=\left[D_{0}+\alpha Q^{\dagger} Q\right] .
$$

In general, with light fermions, $G$ is a very non-local propagator.

We now choose the set $S$ and the decimation parameter $\zeta$ in (2.4) so that in the action

$$
-\bar{\psi} D_{0} \psi-\alpha\left(\bar{\Psi}^{(1)}-\bar{\psi} Q^{\dagger}\right)\left(\Psi^{(1)}-Q \psi\right)
$$

in (2.3) one cancels the nontrivial part of $D_{0}$ :

$$
\bar{\psi}\left[\kappa M-\alpha \zeta\left(\tilde{Q}+\tilde{Q}^{\dagger}\right)\right] \psi=0 .
$$

This is accomplished by setting $\zeta=\kappa / \alpha$, and taking the set $S$ as shown in Fig. 1(a), i.e. $S$, viewed as a subset of $\Lambda$, is the set of the interior sites of the boundary plaquettes of the 2x2-hypercubes that are the elementary d-cells of $\Lambda^{(1)}$. The propagator $G$ for the the original variables $\psi$ in (2.3) is then given by

$$
G^{-1}=1(\alpha)+\frac{\kappa^{2}}{\alpha} \tilde{Q}^{\dagger} \tilde{Q}>0, \quad 1(\alpha)_{n m}=\left\{\begin{array}{cc}
(1+\alpha) \delta_{n m} & n, m \in \Lambda^{\prime} \\
\delta_{n m} & n, m \in \Lambda \backslash \Lambda^{\prime}
\end{array},\right.
$$

and the action in (2.6) from the integration over the original $\psi$ becomes

$$
\bar{\Psi}^{(1)}\left[\frac{\alpha}{1+\alpha}-\kappa^{2} \tilde{Q} G \tilde{Q}^{\dagger}\right] \Psi^{(1)} .
$$

(2.10) is manifestly local on $\Lambda^{\prime}=\Lambda^{(1)} \cup S$. In particular, note that from (2.9), taking $\alpha$ large, interactions induced by $G$ may be expanded in a hopping expansion in $\frac{\kappa^{2}}{\alpha}$ and resummed. In fact, 


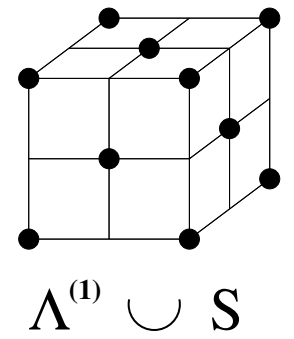

(a)

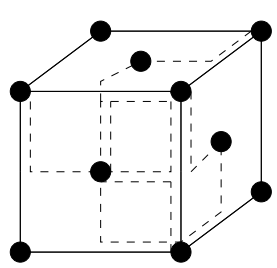

$\Lambda^{(1)} \cup \mathrm{S}$

(b)

Figure 1: (a) The set $S$, as a subset of $\Lambda$, is the set of sites reached from the interior site of each $2 \times 2$ hypercube by displacement by $a$ in each lattice direction; (b) fermions at sites in $S$ interact with fermions on $\Lambda^{(1)}$ and among themselves by 'fat links' consisting of all possible 'staples' of length $2 a$, a subset of which is indicated here by the broken lines.

in the limit $\alpha \rightarrow \infty$, i.e. for the $\delta$-function definition of the blocked variables (cf. above), $G$ becomes ultralocal, and in fact $U$-independent, and the action goes to

$$
\bar{\Psi}^{(1)}\left[1-\kappa^{2} \tilde{Q} \tilde{Q}^{\dagger}\right] \Psi^{(1)} .
$$

After this blocking step we end up with fermions on the boundary of the blocked cube, i.e. a form of exact 'potential moving' fermion blocking albeit on a non-hypercubic lattice. The fermion action is still bilinear in the blocked fermion fields which interact via 'fat' gauge field bonds (Fig. 1(b)). The gauge field bond variables are yet to be blocked. It is important to note that if one starts integrating out gauge field bonds inside each blocked hypercube, four- and higher-fermion interactions will in general be induced (but of course at the scale of the blocked cube). Ultralocality of $G$ in this connection completely avoids potential difficulties from the $\operatorname{Det} G^{-1}$ factor in (2.6) in performing any such accompanying gauge field blocking.

We have devised a fermion blocking step in a general gauge field background that results in a manifestly local action. How to perform successive fermion blocking steps in a fixed gauge field background under the requirement that the action be manifestly local at the scale of each step is an interesting question in its own right. The following is a sufficient condition: for the n-th step implement the above blocking procedure integrating out the fermions on the sites satisfying the conditions:

$$
\begin{aligned}
x_{i} & =2^{n-1} m_{i}, \quad m_{i} \in Z, \quad i=1, \ldots, d, \\
\sum_{i} x_{i} & =2^{n} l+2^{n-1}, \quad l \in Z .
\end{aligned}
$$

Then the n-th step blocked fermions interact again via bilinear interactions through gauge field fat links of maximum length $2^{n}$ (in units of $a$ ). One can actually do better if one allows maximum length $2^{n+1}$ and performs a two-stage blocking of fermions interior to each blocked hypercube. The general lesson, though, is that one cannot achieve a manifestly local blocked fermion action residing on a purely hypercubic lattice, i.e. without some non-empty analog of the set $S$ above (cf. Fig. 1). This seems to accord with previous studies of fermion actions [5]. 


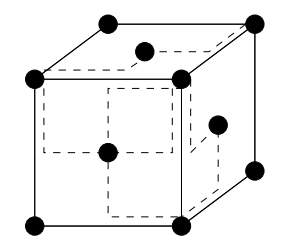

$\Lambda^{(1)} \cup \mathrm{S}$

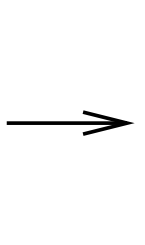

(a)

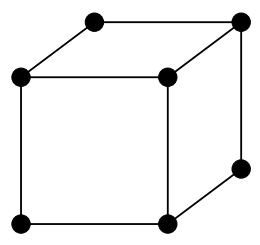

$\Lambda^{(1)}$

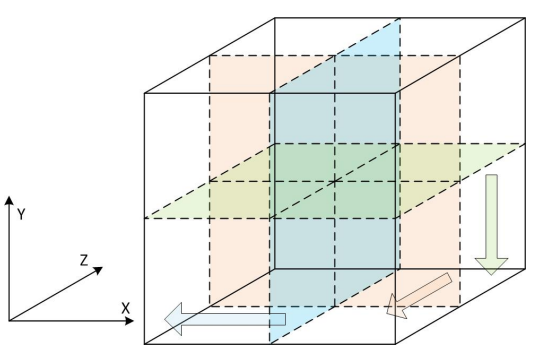

(b)

Figure 2: Approximations: (a) Merge the fermions on $S$ from the exact blocking with the fermions on the hypercube edges while appropriately 'renormalizing' the hopping parameter of the latter, cf. text; (b) standard potential moving approximation for gauge field blocking: move all interior plaquettes symmetrically to the boundary with appropriate weights.

\section{Full RG blocking and approximation schemes}

For the physical questions we are here interested in, i.e. determination of the RG flow and its fixed points, RG blockings of both fermions and gauge fields must of course be implemented. A local blocking step for fermions such as above must then be accompanied by a gauge field blocking step. It is of course possible to define a variety of such blocking transformations, familiar examples of which occur in the literature, in particular, in connection with MCRG. Actually implementing the gauge blocking, however, can generally be done only numerically. In the case of pure gauge theories, MCRG provides a tried method. In the presence of fermions, however, it is not clear exactly how MCRG should be applied. One may, of course, proceed to integrate out the fermions completely, but then the resulting action is very non-local, and application of the ideas underlying the MCRG method is problematic. We will return to these issues elsewhere. Here, in order to get some insight in what to expect, we opt for the modest goal of devising and trying out an approximate simple decimation scheme that can be easily carried out.

To obtain such a scheme we proceed as follows. Eliminate first the fermions on $S$ and compensate by adjusting the hopping parameter of the remaining fermions residing on the hypercubic lattice - this may be viewed as a typical fermion potential moving approximation to get a purely hypercubic action with 'renormalized' gauge interactions (Fig. 2). The 'renormalized' hopping parameter is determined by requiring that some long-distance gauge-invariant fermion correlation function remains invariant under the fermion blocking step. Thus, taking the 2-point function, $\bar{\psi}(x) U\left[\Gamma_{x y}\right] \psi(y)$, (other choices, such a meson-meson correlator, will do equally well) this requirement translates into the exact relation:

$$
\kappa(2 a)=D(\kappa(a)) \kappa(a)^{2},
$$

where

$$
D(\kappa(a))=\frac{\sum_{\mu, v}\left\langle\bar{\psi}_{n}\left(1-\gamma_{\mu}\right)\left(1-\gamma_{v}\right) U\left[C_{n m}\right] U_{\mu}(n) U_{v}(n+\hat{\mu}) \psi_{m}\right\rangle}{\sum_{\mu}\left\langle\bar{\psi}_{n}\left(1-\gamma_{\mu}\right) U\left[C_{n m}\right] U_{\mu}(n) U_{\mu}(n+\hat{\mu}) \psi_{m}\right\rangle} .
$$


(3.1)-(3.2) provide a recursion relation relating the hopping parameters at lattice spacings $a$ and $2 a$, which may, in particular, be solved to obtain the critical hopping parameter. One may then proceed to carry out the gauge field blocking step by whatever method is chosen.

The above approximation allows one to maintain bilinear fermionic interactions at each successive blocking step. This suggests the following considerations. Assume one implements some RG blocking scheme. Denoting by $U^{(n)}, \bar{\psi}^{(n)}, \psi(n)$ the blocked variables at the $n$-th step, then, quire generally, one has

$$
\begin{aligned}
& \int_{\Lambda^{(n)}} D U^{(n)} D \bar{\psi}^{(n)} D \psi^{(n)} \exp \left(-S_{G}^{(n)}-S_{F}^{(n)}\right) \\
= & \int_{\Lambda^{(n+1)}} D U^{(n+1)} D \bar{\psi}^{(n+1)} D \psi^{(n+1)} \exp \left(-S_{G}^{(n+1)}-S_{F}^{(n+1)}\right) .
\end{aligned}
$$

If, furthermore, as after the above approximation, $S_{F}^{(n)}$ and $S_{F}^{(n+1)}$ maintain the same bilinear form on $\Lambda^{(n)}$ and $\Lambda^{(n+1)}$, respectively, then

$$
\exp \left(-S_{G}^{(n+1)}\right)=\int_{\Lambda^{(n)}} D U^{(n)} F\left(U^{(n)}, U^{(n+1)}\right)\left[\frac{\operatorname{Det} S_{F}^{(n)}}{\operatorname{Det} S_{F}^{(n+1)}}\right] \exp \left(-S_{G}^{(n)}\right),
$$

with $F\left(U^{(n)}, U^{(n+1)}\right)$ specifying the blocking relation between $U^{(n)}$ and $U^{(n+1)}$ 's. So if the fermion decimation is sufficiently local, the ratio of determinants in (3.4) can be expected to be reasonably local. Indeed, major part of non-locality is manifestly cancelled in the ratio as seen from the formal expansion of the determinants in terms of gauge field loops: all loops in the expansion of $\ln \operatorname{Det} S_{F}^{(n+1)}$ are contained in that of $\operatorname{Det} S_{F}^{(n)}$. The main contribution to the ratio comes in fact from loops inside each blocked hypercube. This suggest that one may approximate it by just such a set.

\subsection{Simplest RG implementation model}

These considerations lead us to an approximate implementation of RG decimations of the full system of fermions and gauge fields given by the following recipe.

After a fermion blocking step, apply the approximation depicted in Fig. 2(a) combined with (3.1)-(3.2). Then the gauge field action on the spacing $2 a$ lattice is given by (3.4). As just explained, after the cancellation of the denominator by the numerator in the determinant ratio expressed in terms of gauge field loops, we approximate the remainder by the contributions of loops interior to each blocked hypercube - in particular, by just the single plaquette loop weighted by a parameter $\eta$. (One may of course consider approximations involving more interior loops, e.g. $2 \times 2$ loops, and more decimation parameters.) It remains to specify and perform a gauge field blocking over each hypercube. This we do by standard potential moving decimation: every interior plaquette is moved to the hypercube boundary with the appropriate weights $2^{d-2}$ (Fig. 2(b)). Expanding all quantities in group characters one thus obtains the recursion relations relating the coefficients in the character expansion of the 1.h.s of (3.4) to the coefficients of the character expansions of the determinant and action factors in the integrand on the r.h.s. The procedure may then be iterated.

This scheme depends only on two quantities: the parameter $\eta$, and, at each blocking step, the critical $\kappa\left(2^{n} a\right)$. We have applied it to $S U(2)$ and $S U(3)$. The results are shown in Table $1 . N_{\mathrm{L}}$ and $N_{\mathrm{U}}$ denote the number of flavors at which an IR fixed point first appears and that at which 


\begin{tabular}{|l|l|l|}
\hline & $N_{\mathrm{L}}$ & $N_{\mathrm{U}}$ \\
\hline $\mathrm{SU}(2)$ fund & 9 & 11 \\
SU(2) adjoint & 2 & 3 \\
SU(3) fund & $11-12$ & 17 \\
$\mathrm{SU}(3)$ sextet & 2 & 4 \\
\hline
\end{tabular}

Table 1: Conformal window lower and upper bounds as obtained from the simple blocking recursion relations described in the text.

asymptotic freedom is lost, respectively. The parameter $\eta$ was fixed by the value $N_{\mathrm{U}}$ for the upper bound of the conformal window for $S U(2)$ fundamental representation fermions. This decimation parameter could in principle be tuned independently in each case, but it turns out that once set by the top entry in the second column, no further adjustment need be made to produce the rest of the entries with the correct $N_{\mathbf{U}}$ values. Having fixed the parameter $\eta$ the only uncertainties in practice are in obtaining the critical $\kappa$ values. They were obtained from estimates from (3.2) and/or values from simulations reported in the literature [6]. The resulting recursion relations for the character coefficients can then be run, very cheaply, to essentially arbitrary accuracy. It is noteworthy that such an approximate decimation scheme can already produce results such as shown in Table 1.

\section{Conclusions - Outlook}

We have explored locality-preserving RG blocking schemes for light fermions. We saw that blockings maintaining locality in an arbitrary gauge field background can be devised, but they generically require non-hypercubic blocked fermion actions. Such schemes may prove useful in investigating a variety of interesting issues (e.g. the 'rooting' of fermion determinants). Incorporation in MCRG procedures to perform full (fermion plus gauge field) RG blocking would seem to provide the most direct and reliable way for elucidating IR phase structure with varying fermion content. Relatively crude but inexpensive schemes already provide, as we saw, rather encouraging results.

This research was partially supported by NSF PHYS-0852438.

\section{References}

[1] For a recent review, see L. Del Debbio, PoS LATTICE 2010 004; PoS LATTICE 2010 and PoS LATTICE 2011 (these proceedings) provide a rather complete picture of the current state of the field.

[2] Anna Hasenfratz, arXiv:1106.5293 [hep-lat); S. Catterall, L. Del Debbio, J. Giedt and L. Keegan, arXiv:1108.3794 [hep-ph].

[3] T. Balaban, M. O’Carroll and R. Schor, Comm. Math. Physics. 122, 233 (1989).

[4] Y. Shamir, Phys. Rev. D 71, 034509 (2005).

[5] T. DeGrand, Phys. Rev. D 58, 094503 (1998) and references therein.

[6] Y. Shamir, B. Svetitsky and T. DeGrand, Phys. Rev. D 78, 031502 (2008); S. Catterall et al, JHEP 0811:009 (2008); Y. Ywasaki et al, Phys. Rev. D 69, 014507 (2004). 